\title{
Ten years of RELEA: Achievements and challenges for astronomy education development
}

\author{
P. S. Bretones ${ }^{1}$, L. C. Jafelice ${ }^{2}$ and J. E. Horvath ${ }^{3}$ \\ ${ }^{1}$ Departamento de Metodologia de Ensino, Universidade Federal de São Carlos, São Carlos, \\ Brazil \\ ${ }^{2}$ Departamento de Fsica, Universidade Federal do Rio Grande do Norte, Natal, Brazil \\ ${ }^{3}$ Instituto de Astronomia, Geofísica e Ciências Atmosféricas, Universidade de São Paulo, São \\ Paulo, Brazil
}

When an area of education, and more particularly the research within this area, is aimed to development, one of the basic requirements is the existence of a regular publication that accounts for the scientific production in that area. This study aims to analyze 10 years of Latin-American Journal of Astronomy Education (RELEA) [http://www.relea.ufscar.br/].

The 75 articles published in 18 editions were analyzed. The acceptance rate of the articles is $60.2 \%$, while the refereeing time average is about 14 days, the annual average is 7.5 articles and the issue average is 4.2 articles. A large majority of the articles originated from Brazil (61-81.3\% of the published). The rest of the works were contributed by Argentina (6-8.0\%), Uruguay (2-2.7\%), USA (3-4.0\%), Spain (2-2.7\%), and New Zealand (1-1.3\%). Concerning the school grade level or public outreach addressed in the papers, the largest percentage were related to university education $(28.0 \%)$ and to high school $(28.0 \%)$, while $25.3 \%$ of works deal with unspecified school grade level. Concerning the focus of study on education there is a predominance of papers on learning and teaching of astronomy education (34.7\%), followed by studies of students understanding $(17.3 \%)$ and development and discussion of teaching materials $(13.3 \%)$. Other works were found related to: Teacher's education $(8.0 \%)$, studies on teachers understanding $(8.0 \%)$, curricular discussions/programs in astronomy (6.7\%), studies of history and philosophy of science $(5.3 \%)$, non-school programs $(4.0 \%)$. With respect to the contents, most studies do not deal with specific topics in astronomy and we labeled these as General (33.3\%). Studies of Sun-Earth-Moon System (26.7\%) and Solar System (18.7\%) are the second and the third largest groups. Less frequent are studies which include history of astronomy, positional astronomy and constellations as the main. These results present trends and shortcomings of the production.

This journal is now consolidated but the number of articles is still relatively small probably because of the lack of publishing tradition in the area. Its future challenges include how to increase the number of published articles; specially from Latin American countries, and how to bring in the issues and subjects not addressed until now; training of the community for a better quality of the submitted articles and the recent restructure of the Editorial Board. It is also considered the possibility of encouraging graduate studies, new lines of research in astronomy education, and dissemination of material in universities and schools for teachers and students. 
Finally, existing future possibilities are given by the IAU development programs. For example, more article submission from Portuguese-speaking countries with the support of Regional Nodes and Language Expertise Centers, advertising by the OAD and opportunities for volunteer IAU members and global projects for the development of astronomy education. 